\title{
Flow cytometry and fluorescence-activated cell sorting in plants: the past, present, and future
}

\begin{abstract}
David W. Galbraith
Introduction: Flow cytometry and cell sorting are powerful technologies for examining the molecular, genetic, and physiological properties of individual cells.

Objective: The objective of this article is to provide a historical survey of the development of flow cytometry and cell sorting for use with higher plants, a summary of the state of art at the present day, and a prediction of where the field might progress over the coming years.

Methods: Adapting flow cytometry and sorting for use with higher plants requires the production of single cell suspensions, or suspensions of subcellular organelles. It also requires identification of methods for fluorescence labeling of the cells or organelles of interest, such that they can be usefully analyzed and sorting. These methods are identified and outlined.

Results and conclusions: Recent advances in molecular and biotechnological methods, platforms, and instrumentation, combined with flow cytometry and sorting, provide increasingly powerful analytical tools for exploring the components and structure of regulatory networks governing plant growth and development, and the interactions of plants with their environments. They also will be invaluable in cataloguing the individual species that comprise the biological diversity of flowering plants.
\end{abstract}

Key words: flow cytometry, cell sorting

Flow cytometry is a technological platform that was originally developed for analysis of mammalian blood cells, which comprise a natural suspension of individual cells. These are constrained to flow within a liquid stream, through the focus of an intense light source. Each cell both scatters and absorbs light. If labeled in some way with fluorescent molecules, these cells also emit signals in the form of fluorescence. The flow cytometer quantifies and records these light scatter and fluorescence signals as parametric properties of each cell. The properties are dictated by the specificities of the fluorochromes, of which a very large number are available. These can be placed into two general classes: fluorochromes that are endogenous to the cells, and fluorochromes that are added exogenously. The former can be subdivided into (i) naturally occurring pigments, such as chlorophyll, and other naturally fluorescent small molecules within the cell, and (ii) endogenous fluorochromes that are produced transgenically, for example the fluorescent proteins. Fluorochromes added exogenously to cells can be also subdivided into (i) the group of small synthetic molecules that fluoresce only after specifically binding to

\footnotetext{
Corresponding author:

University of Arizona, School of Plant Sciences and Bio5 Institute for Collaborative Bioresearch, Tucson, Arizona 85721, USA

Telephone: +1 (520) 6219153; fax: +1 (520) 6264824

galbraith@arizona.edu
}

cellular components, for example, 4',6-diamidino2-phenylindole (DAPI) for DNA, and (ii) the group of fluorescent molecules that lack innate specificity for cellular components, instead being used for covalent reaction with other macromolecules having defined specificities, for example fluorescein isothiocyanate and antibodies.

Clever combination of multiple sources of laser illumination, optical filters, and compensation circuits has allowed analysis of as many as 22 different independent parameters of mammalian cells (1). Flow cytometric datasets are conventionally displayed as multiparametric frequency distributions, with gates placed within this distributions being used to identify subsets of cells of specific interest. Visualization beyond 3-dimensions is not conceptually intuitive, so analysis of datasets of high dimensionality typically relies on setting multiple logical gates to progressively define subsets of cells with defined characteristics.

Given that flow cytometry generally allows recognition of subsets of cells having distinctive optical properties, cell sorting was developed to allow the selective purification of these subsets for further analysis. Most cell sorters operate using a jet-in-air configuration, in which the natural tendency of surface tension to convert liquid jets into droplets is precisely constrained in time and space by 
imposition of a periodic disturbance on the flow tip (figure 1). This means that the time delay between detection of an object, by passage through the laser focus, and entry into the last-attached droplet below the flow tip, is a fixed, constant value. At this point of entry, therefore, a charge can be placed on the flow stream, which is retained on the surface of the droplet after it detaches. The droplet then is deflected for collection by passage through a zone containing a fixed electrostatic field. Since droplet charging can be rapidly switched, sorting at high rates can be achieved, and this allows collection even of cells that are rare within the starting suspension.

Since higher plants, in general, do not comprise suspensions of single cells, they are not naturally compatible with flow cytometric instruments. Instead, methods are required to produce plant derived suspensions that can pass through the flow tips. Two general strategies are available: the first is to employ protoplasts for flow cytometry and sorting. Protoplasts are produced by enzymatically dissolving the cell wall under slightly hypertonic conditions. They are living cells, albeit fragile ones, and under appropriate conditions of culture can be stimulated to resynthesize the cell wall, enter into cell division, and even, for selected examples, regenerate into whole plants. The second strategy involves applying flow cytometry and sorting to subcellular organelles, particularly nuclei, produced through homogenization of the plant tissues. Although evidently not permitting analysis of living cells, this approach has considerable relevance currently in genomics and, in the future, most likely in proteomics. Over the last 30 years, the development of flow cytometry and sorting for plants has advanced steadily, and its popularity has recently increased rapidly.

\section{The past}

The first report of the application of flow cytometry to the study of higher plants appeared in the early 1970's, but attracted little attention from the scientific community (2). This work involved incubation of acetic acid/ethanol-fixed root tips of Vicia faba in a mixture of pectinase and pepsin. The resultant suspension was stained with ethidium bromide (EB), and the fluorescence characteristics of the objects within this suspension determined using a Phywe-ICP 11 flow cytometer. Two distinct peaks of fluorescence were observed, which were interpreted as corresponding to nuclei derived from $\mathrm{G} 0 / \mathrm{G} 1$ and $\mathrm{G} 2$ of the cell division cycle.
This interpretation is likely correct, despite the fact that treatment of fixed roots with pectinases and pepsin is not recommended as a method for isolating plant nuclei. Although the suspension in actuality comprised a mixture of nuclei, intact cells,

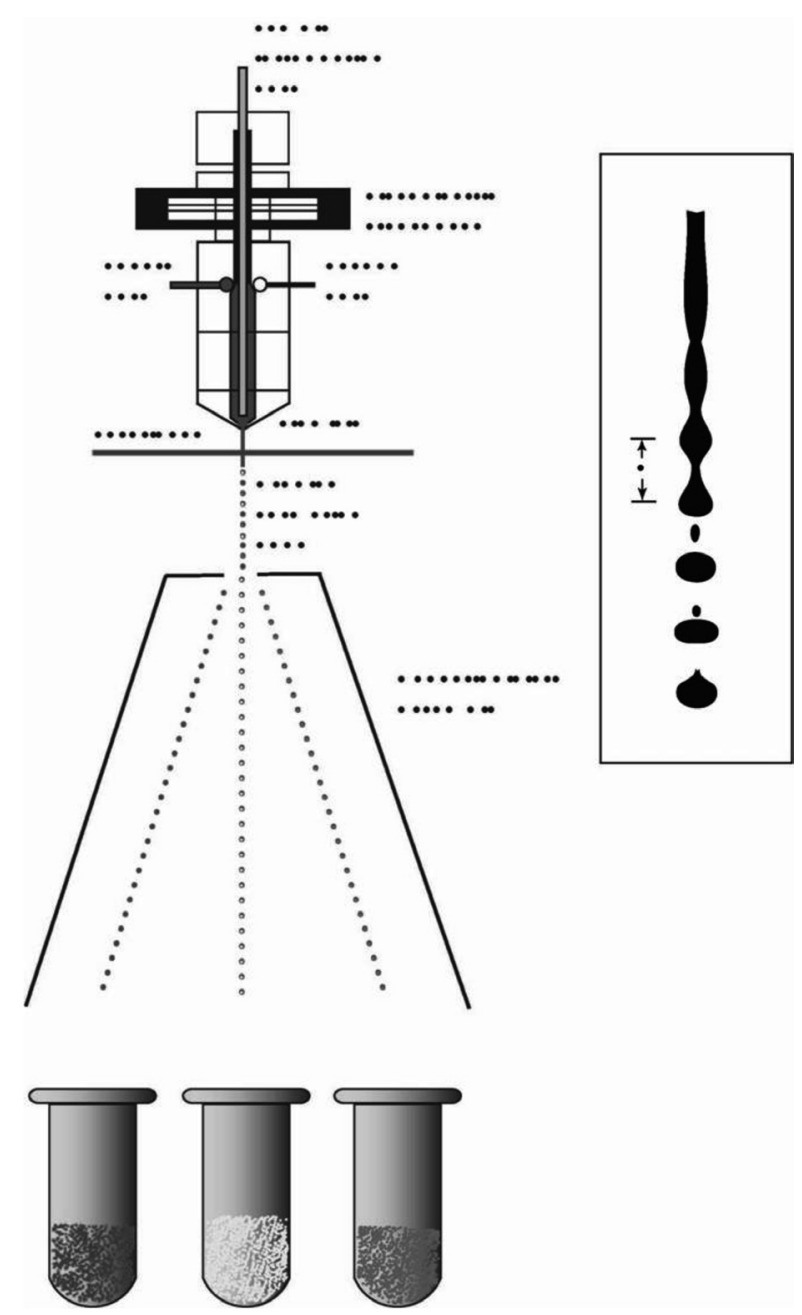

Figure 1. Schematic illustration of the flow cell of a typical jetin-air flow cytometer/cell sorter. The sample is injected into the center of the flow stream based on slight differences in pressure between the sample and sheath lines. Hydrodynamic focusing then ensures the samples are centered within the stream emerging from the flow tip. Droplet formation is synchronized by the action of the piezoelectric actuator attached to the flow cell. A charge applied to the flow stream with an appropriate time delay ensures droplets enclosing the desired cell carry residual charge and are deflected as they pass through the electrostatic field. Up to four different deflection amplitudes are possible in most commercial instruments, two positive and two negative; a two-way sort is illustrated here, with unwanted cells being undeflected.

Insert: The process of formation of droplets is a result of surface tension; the undulation wavelength $(\lambda)$ is a function of the flow stream velocity, and the frequency of actuation of the piezo. Droplet formation requires that $\lambda$ be greater than the diameter of the liquid jet (see references 8 and 32 for a full discussion). 
and miscellaneous subcellular debris, the fact that distinctive peaks were observed was most likely a consequence of the choice for analysis of Vicia faba, which has a very large genome in comparison to other crop species. These would be expected to produce readily detectable fluorescence signals with flow cytometry, and these bright signals would be also be somewhat resistant to optical distortion. The use of EB staining without an accompanying ribonuclease step, which results in fluorescence emission from both nuclear RNA and DNA, would also serve to enhance the intensity of the signals. These technical issues imply that the methods cannot be usefully generalized to other higher plant species, and provide further insight into the reasons that this work was not recognized and adopted by the scientific community.

Use of flow cytometry for analysis of plant genome sizes and cell cycle status entered the scientific mainstream upon development of a simple chopping procedure for preparation of nuclei (3). This involves minimal manipulation of the biological materials -the homogenate is simply filtered to remove large debris that would otherwise clog the flow tips, and the nuclei stained using DNA-specific fluorochromes. The advantages of the method are speed of sample preparation, its general applicability across the flowering plants (4) and across tissue types (5), and the value of the different types of applications for which DNA genome size measurements are required. These include identification of novel species, cytotypes, and hybrids, detection of agriculturally-relevant ploidy classes, and applications within tissue culture and in vitro plant manipulation and propagation. A comprehensive discussion of applications can be found in Galbraith, et al. (6).

Analyses of nuclear DNA contents using flow cytometry result in uniparametric datasets, in which the amount of fluorescence linearly scales with DNA content. The first applications of flow cytometry to single plant cells also involved uniparametric measurements. As previously discussed, it is first necessary to make single cell suspensions, which either are in the form of protoplasts, or exploit natural single cell situations, such as pollen. Protoplasts and pollen frequently are close to or even larger than the flow cell tips typically installed on flow sorters, hence requiring the use of large flow tips (diameters of up to $200 \mu \mathrm{m}$ ). Modifications to the sheath pressures and piezo actuation frequencies are required for stable large particle sorting (7), but protoplasts do survive passage through the flow cytometer and the process of sorting and can regenerate into complete plants (8).

Multiparametric flow analyses using plants started with the concept of employing flow sorting for the isolation of rare heterokaryons produced through protoplast fusion (9). In this case, simple covalent labeling of the two sets of parental protoplasts, produced from different species, was done using fluorescein isothiocyanate (FITC) and rhodamine isothiocyanate (RITC). Protoplast fusion was induced by chemical treatment, and the hybrid protoplasts sorted based on the presence of both FITC and RITC. Somatic hybrid plants were then regenerated from the sorted protoplasts.

Multiparametric analysis and flow sorting of protoplasts was subsequently applied for the characterization of molecular regulation at the level of different cell types, based on the ability to distinguish leaf cells, mesophyll and epidermis, according to chlorophyll content (10). However, a true explosion of interest in this technology first required emergence of the class of in vivofluorescent protein markers, for which the founding member and prototype is the green fluorescent protein (11), and the development of Arabidopsis as a model plant genetic and genomic system, including complete sequencing of the nuclear genome, and development of facile transformation procedures. Transient and transgenic GFP expression is readily detected in plant protoplasts and in isolated nuclei using flow cytometry $(12,13)$, and regulating GFP expression with cell type-specific sequences leads to sorting of protoplasts prepared from transgenic plants that represent these cell types (14). Alternatively, GFP can be targeted to the nucleus, and fluorescent nuclei sorted $(15,16)$. In either case, global expression profiling platforms can then be used to define classes of transcripts that are up and down regulated in concentrations within specific cell types. Further information emerging from the latter approach concerns the ploidy status of specific cell types within complex organs (15).

Other applications of flow cytometry and sorting as applied to protoplasts are described in a recent review (17).

\section{The present}

Use of flow cytometry for genome size measurements is expanding further based on the development of low-cost, portable instruments such as the Accuri C6 (Galbraith, 2009), the Partec 
CyFlow Ploidy Analyzer, and the recently-released Attune Acoustic Focusing Cytometer. The flowering plants are estimated to comprise around 500,000 species, and it now appears practical, using portable instruments such as these, to measure the genome sizes of all plant species, as a preliminary to full genome sequencing at some depth of coverage. The importance of naming species has been noted, particularly given the increasing rate of species extinction due to human population growth and resource exploitation (18).

Use of flow cytometry and sorting for characterization of cell type-specific gene expression continues to expand. This includes a variety of cell types found within the root (19-22), and in the shoot apex (23). Flow sorting of protoplasts was recently extended to allow measurement of auxin levels within specific cell types (24). Remarkably, protoplasts appear to preserve levels of metabolites that reflect cellular concentrations in situ, although correction for cytoplasmic volume changes are, of course, required.

Further development of FP markers includes recent reports from two groups, extending the palette of colors of these markers that can be usefully expressed in plants $(25,26)$. Bargmann and Birnbaum (27) have illustrated the use of biparametric flow analysis and sorting combined with transfection using pairs of FPs, for selective examination of signal transduction pathways and their response to perturbation.

\section{The future}

The analytical capability of flow cytometry and sorting extends to single cells and further development of downstream methods to accommodate single cell measurements represents an active area of research that will continue to expand. This invokes stochasticity and involves measurement of between-cell variation, as pioneered in yeast using flow cytometry $(28,29)$. Variation of course can be a function of transcription, translation, and protein degradation, or any combination of these factors. The general ability to be able to measure any property within a single plant cell represents an ultimate goal of this type of approach, as is already possible for whole genome transcriptomics in mammalian systems (30). Extension of single cell measurements to epigenetic marks, the complement of small RNAs, the proteome, and the metabolome represent significant technical challenges, but I am optimistic that these will be solved over the coming decade.
In terms of FP technologies, it should be simple to adapt multiparametric flow cytometry and sorting platforms for multicolor analysis of FPs in plant protoplasts and nuclei, as previously demonstrated for mammalian cells (31). Further emergence of brighter and more-stable FPs also appears highly likely. Extending plant flow cytometric measurements to higher dimensions should also become possible, as demonstrated for mammalian systems (1), and incorporating both existing and novel fluorochromes.

In terms of instrumentation, further decreases in instrument costs can be anticipated, based in part on advances in microfluidics manufacturing, which may lead to novel flow cytometer design configurations, and in part on the general decrease in the component costs of conventional flow cytometers (laser costs being particularly notable). It is anticipated that the cost of sort-capable flow cytometers will follow the trend of decreasing cost already seen for non-sort capable instruments. The ability to chart genome sizes world-wide will be particularly facilitated by further decreases in instrument costs and in platform sizes, and integration of these instruments into satellite-based localization and communication systems will greatly simplify data collection, cataloging, and archiving.

\section{Summary and conclusions}

Flow cytometry and fluorescence-activated sorting are mature technologies that have been fully integrated into plant biology. Their impact has been profound both in terms of basic discovery of knowledge and in terms of providing measurements of direct relevance to agriculture, crop production and protection, and the environment. We look forward to the broadening of this impact as affordable instruments are increasingly deployed within plant laboratories, and as novel applications continue to be developed.

\section{Acknowledgments}

I thank Georgina Lambert for valuable technical assistance associated with operating the various flow cytometers in my laboratory.

\section{Conflicts of interest and financial support statements}

The author declares no conflicts of interest.

This work was supported in part by grants from the National Science Foundation and the United States Department of Agriculture. 


\section{References}

1. Chattopadhyay PK, Hogerkorp CM, Roederer M. A chromatic explosion: the development and future of multiparameter flow cytometry. Immunology. 2008;125:441-9.

2. Heller FO. DNA measurement of Vicia faba L. with pulse cytophotometry. Ber Dtsch Bot Ges. 1973;86:437-41.

3. Galbraith DW, Harkins KR, Maddox JR, Ayres NM, Sharma DP, Firoozabady, E. Rapid flow cytometric analysis of the cell cycle in intact plant tissues. Science. 1983;220:1049-51

4. Galbraith DW. Simultaneous flow cytometric quantification of plant nuclear DNA contents over the full range of described angiosperm 2C values. Cytometry. 2009;75A:692-8.

5. Sliwinska E, Pisarczyk I, Pawlik A, Galbraith DW. Measuring genome size of desert plants using dry seeds. Botany. 2009;87:127-35.

6. Galbraith DW, Bartos J, Dolezel J. Flow cytometry and cell sorting in plant biotechnology. In: Sklar LA, editor. Flow cytometry in biotechnology. Oxford: Oxford University Press; 2005. p. 291-322.

7. Harkins KR, Galbraith DW. Flow sorting and culture of plant protoplasts. Physiol Plant. 1984;60:43-52.

8. Harkins KR, Galbraith DW. Factors governing the flow cytometric analysis and sorting of large biological particles. Cytometry. 1987;8:60-71.

9. Afonso $\mathrm{CL}$, Harkins $\mathrm{KR}$, Thomas-Compton M, Krejci A, Galbraith DW. Production of somatic hybrid plants through fluorescence-activated sorting of protoplasts. Nat Biotechnol. 1985;3:811-6.

10. Harkins KR, Jefferson RA, Kavanagh TA, Bevan MW, Galbraith DW. Expression of photosynthesis related gene fusions is restricted by cell type in transgenic plants and in transfected protoplasts. Proc Natl Acad Sci USA 1990;87:816-20.

11. Chalfie M, Tu Y, Euskirchen G, Ward WW, Prasher DC. Green fluorescent protein as a marker for gene expression. Science. 1994;263:802-5.

12. Sheen J, Hwang S, Niwa Y, Kobayashi H, Galbraith DW. Green fluorescent protein as a new vital marker in plant cells. Plant Journal. 1995;8:777-84.

13. Grebenok RJ, Lambert GM, Galbraith DW. Characterization of the targeted nuclear accumulation of GFP within the cells of transgenic plants. Plant Journal. 1997;12:685-96.

14. Birnbaum K, Shasha DE, Wang JY, Jung JW, Lambert GM, Galbraith DW, et al. A gene expression map of the Arabidopsis root. Science. 2003;302:1956-60.

15. Zhang CQ, Gong FC, Lambert GM, Galbraith DW. Cell type-specific characterization of nuclear DNA contents within complex tissues and organs. Plant Methods. 2005;1:7.

16. Zhang CQ, Barthelson RA, Lambert GM, Galbraith DW. Characterization of cell-specific gene expression through fluorescence-activated sorting of nuclei. Plant Physiol. 2008;147:30-40
17. Galbraith DW. Protoplast analysis using flow cytometry and sorting. In: Dolezel J, Greilhuber J, Suda J, editors. Flow cytometry with plant cells. New York: Wiley-VCH; 2007. p. $231-50$.

18. Jackson PW, Kennedy K. The global strategy for plant conservation: a challenge and opportunity for the international community. Trends Plant Sci. 2009;14:578-80.

19. Nawy T, Lee J-Y, Colinas J, Wang JY, Thongrod SC, Malamy JE, et al. Transcriptional profile of the Arabidopsis root quiescent center. Plant Cell. 2005;17:1908-25.

20. Lee JY, Colinas J, Wang JY, Mace D, Ohler U, Benfey PN. Transcriptional and post-transcriptional regulation of transcription factor expression in Arabidopsis roots. Proc Natl Acad Sci USA. 2006;103:6055-60.

21. Brady SM, Orlando DA, Lee JY, Wang JY, Koch J, Dinneny JR, et al. A high-resolution root spatiotemporal map reveals dominant expression patterns. Science. 2007;318:801-6.

22. Dinneny JR, Long TA, Wang JY, Jung JW, Mace D, Pointer $\mathrm{S}$, et al. Cell identity mediates the response of Arabidopsis roots to abiotic stress. Science. 2008;320:942-5.

23. Yadav RK, Girke T, Pasala S, Xie MT, Reddy V. Gene expression map of the Arabidopsis shoot apical meristem stem cell niche. Proc Natl Acad Sci USA. 2009;106:4941-6.

24. Peterson SV, Johansson Al, Kowalczyk M, Makoveychuk A, Wang JY, Moritz, T et al. An auxin gradient and maximum in the Arabidopsis root apex shown by high-resolution cellspecific analysis of IAA distribution and synthesis. Plant Cell. 2009;21:1659-68.

25. Nelson BK, Cai X, Nebenführ A. A multicolored set of in vivo organelle markers for co-localization studies in Arabidopsis and other plants. Plant Journal. 2007;51:1126-36.

26. Geldner N, Denervaud-Tendon V, Hyman DL, Mayer U, Stierhof YD, Chory J. Rapid, combinatorial analysis of membrane compartments in intact plants with a multicolor marker set. Plant Journal. 2009;59:169-78.

27. Bargmann BOR, Birnbaum KD. Positive fluorescent selection permits precise, rapid, and in-depth overexpression analysis in plant protoplasts. Plant Physiology. 2009;149:1231-9.

28. Raser JM, O'Shea EK. Control of stochasticity in eukaryotic gene expression. Science. 2004;304:1811-4

29. Newman JRS, Ghaemmaghami S, Ihmels J, Breslow DK, Noble M, DeRisi JL, et al. Single-cell proteomic analysis of $S$. cerevisiae reveals the architecture of biological noise. Nature. 2006;441:840-6.

30. Tang FC, Barbacioru C, Wang Y, Nordman E, Lee C, Xu N, et al. mRNA-Seq whole-transcriptome analysis of a single cell. Nature Meth. 2009;6:377-U86.

31. Hawley TS, Telford WG, Ramezani A, Hawley RG. Fourcolor flow cytometric detection of retrovirally expressed red, yellow, green, and cyan fluorescent proteins. Biotechniques. 2001;30:1028-34

32. Galbraith DW, Lucretti S. Large particle sorting. In: Radbruch A, editor. Flow cytometry and cell sorting. Second edition. Berlin: Springer-Verlag; 2000. p. 293-317. 
